\title{
Automated Detection of Left Ventricle in 4D MR Images: Experience from a Large Study
}

\author{
Xiang Lin ${ }^{1}$, Brett R. Cowan ${ }^{2}$, and Alistair A. Young ${ }^{1}$ \\ ${ }^{1}$ Bioengineering Institute, University of Auckland, New Zealand \\ ${ }^{2}$ Center for Advanced MRI, University of Auckland, New Zealand \\ $\{\mathrm{x}$. lin, b.cowan, a.young\}@auckland.ac.nz
}

\begin{abstract}
We present a fully automated method to estimate the location and orientation of the left ventricle (LV) in four-dimensional (4D) cardiac magnetic resonance (CMR) images without any user input. The method is based on low-level image processing techniques incorporating anatomical knowledge and is able to provide rapid, robust feedback for automated scan planning or further processing. The method relies on a novel combination of temporal Fourier analysis of image cines with simple contour detection to achieve a fast localization of the heart. Quantitative validation was performed using $4 \mathrm{D}$ CMR datasets from 330 patients (54024 images) with a range of cardiac and vascular disease by comparing manual location with the automatic results. The method failed on one case, and showed average bias and precision of under $5 \mathrm{~mm}$ in apical, mid-ventricular and basal slices in the remaining 329. The errors in automatic orientation were similar to the errors in scan planning as performed by experienced technicians.
\end{abstract}

\section{Introduction}

We investigated methods for the robust, accurate and fully automatic identification of heart location and orientation from CMR examinations. The method is targeted for clinical applications and must therefore be fast, efficient and reliable. It should be able to return the location, orientation and approximate contours of the LV in the absence of any user input. The method is expected to have two important applications. Firstly, the detected LV contours could be used as input to higher level segmentation methods and deformable model based analysis. Secondly, the method can be used to speed up the acquisition time by facilitating the fully automatic planning of CMR examinations.

Segmentation of the LV in CMR images is important for quantitative assessment of the function of the heart and many automatic approaches at different image processing levels have been proposed for this problem. Low level techniques depending on the local intensity characteristics are fast but lack robustness. $A$ priori knowledge can be incorporated in deformable model-based methods, however, the efficiency and robustness of the methods is heavily dependent on the initial contours or models. Most semi-automatic methods like 1 require manual initialization. Fully automatic algorithms can be found but many of these 
are computation-intensive [2] or lack of a wide range of clinical validation [3]. One [4] has been validated in 121 cases but assumes that the location of the heart is approximately at the center of the MR image. Specialized methods have also been proposed for tagged images [5] or perfusion images [6], however their application to patients with a wide range of clinical disease is unclear.

Automated CMR image planning has been proposed for speeding up scan time 7, 8. The core requirement is for a fast and accurate calculation of the threedimensional (3D) position and orientation of the LV. A deformable template based method was addressed in [7], which estimated the LV axis by fitting many feature points of major organs in the thorax to the localizer images. However, the method was computation-intensive. To avoid this problem, 8, proposed another method which employed a priori knowledge of the average LV direction to speed up their procedure. The localizer images were then segmented by thresholding and both the LV and RV were localized by comparison of the morphologic characteristics of the candidate objects. However, in our experience clinical image variability compromises the robustness of this method.

We relied on simple methods to automatically estimate heart location and orientation, in order to provide rapid feedback to higher level processes. The assumptions of our method are listed below. Any cases which violate these assumptions (eg congenital heart disease in which the LV and RV are transposed) would not be expected to be solved by our method.

1) The heart is the only large organ in the thorax with a spatial fundamental frequency equivalent to the cardiac cycle.

2) The orientation of the heart is similar across a wide variety of (non-congenital) cardiac diseases. (This assumption is validated below.)

3) The short axis (SA) slices are ECG gated and have been planned approximately orthogonal to the long axis of the LV. (We show that this assumption is not restrictive in practice.)

4) The positions of LV in adjacent slices are spatially and temporally coherent.

5) The septal myocardium is close to the centroid of the heart and has the LV and RV blood pools on each side. The boundary between the LV blood pool and septal myocardium is not degraded by large papillary muscles or trabeculations, which are typically not expected in this area anatomically.

The reminder of the paper is organized as follows. In Sect. 2, we describe the details of our method. In Sect. 3, we present the results from 330 patients recruited from $10 \mathrm{MR}$ centers world-wide. Conclusion and discussion is provided in Sect. 4 .

\section{Method}

Our method is based on the novel combination of the Fourier transform (FT) in the temporal domain with a priori orientation and shape information in space. The FT is employed to calculate an average image (DC) and a first harmonic (H1) magnitude image for each slice. Even in severely diseased hearts, this 
method successfully identifies a large portion of the heart in most cases. The FT output is then used to derive a region of interest (ROI) and threshold which robustly delineates the LV. This four step process is summarized below:

1) Organize the frames slice by slice and apply the FT over time to obtain the DC and H1 images for each slice (Sect. 2.2).

2) Compute a ROI for each slice and the centroid of the whole heart from the H1 images (Sect. 2.3).

3) Find a pixel on the septal myocardium and compute the threshold level of the blood in the DC images (Sect. 2.4).

4) Threshold the DC images and locate the LV on all slices (Sect. 2.5).

\subsection{Patient Data and Ground Truth}

We examined 330 patients with cardiac and vascular disease enrolled in the CMR substudy to ONTARGET [9]. The patients had a range of disease histories: 294 had coronary artery disease, 46 had peripheral arterial disease, 111 had diabetes, 202 had hypertension and 192 had myocardial infarction with the total exceeding the number of patients due to multiple diagnoses. The patients were recruited in six countries and imaged using standard SSFP cardiac cine sequence on Siemens, Philips and GE scanners. Either prospectively or retrospectively gated cine images were acquired in six equally spaced SA locations from apex to base. Typical imaging parameters were TR / TE / flip / FOV $=30 \mathrm{~ms} / 1.6 \mathrm{~ms} / 60^{\circ} / 360 \mathrm{~mm}$, slice thickness $6 \mathrm{~mm}$, image matrix $256 \times 208$. There were typically 25 temporal frames per slice, depending on heart rate. All cines were acquired during breath-hold of 8-15 seconds duration.

The ground truth for the heart location and orientation was determined manually by two experienced users operating independently on the end-diastolic phase images. The $3 \mathrm{D}$ orientation of the LV long axis $V_{x}$ was defined by two points located in the middle of the LV blood pool at apex and base (Fig. 1] and 1 $1 \mathrm{~b}$ ). The orientation of the RV $V_{y}$ was defined by the centroid of the RV insertion points defined on each SA slice (Fig. 1 ). The remaining axis $V_{z}$ was oriented posterior to create a right handed coordinate system (Fig. 11d) [10]. The average directions $\bar{V}_{x}, \bar{V}_{y}$ and $\bar{V}_{z}$ of all cases were pre-computed for use in the automated method below.

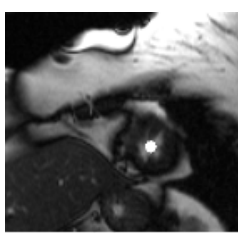

(a)

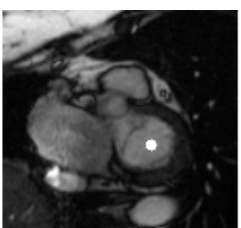

(b)

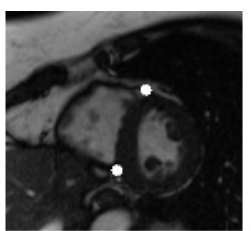

(c)

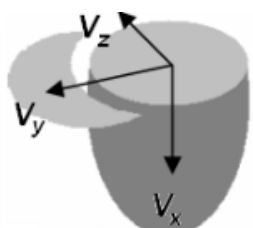

(d)

Fig. 1. Manual definition of heart orientation 


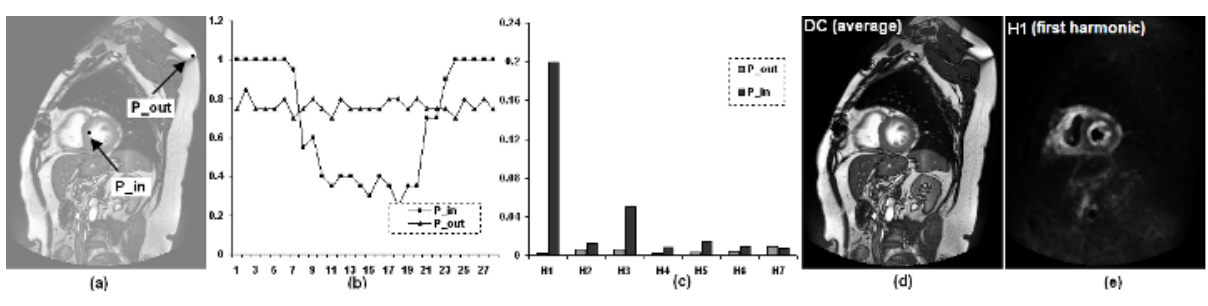

Fig. 2. Reasons for using FT over time sequence

\subsection{Fourier Transform over Time}

The heart is the only large structure in the thorax which has substantial motion with a frequency given by the heart rate, and this characteristic makes the heart distinguishable by analyzing the change in the intensity values over time at each pixel position. Fig. 2 $\mathrm{b}$ shows typical pixel intensities through time. $P_{\text {in }}$ is a pixel at the boundary between the LV blood pool and the septal myocardium. Its intensity value changes through a large range over time. $P_{\text {out }}$ is also located close to the boundary of two different structures but is relatively static. Previously the standard deviation of the pixel values has been used to locate the heart [11,12, however, we found that in about $20 \%$ of cases the standard deviation images were contaminated by excessive high frequency noise. We therefore computed the Fourier transform (Fig. 25) and used the DC component (average image, Fig. 2d) and the H1 image (Fig. 2e) only. This method provides excellent delineation of the cardiac structures, as well as great vessels such as the aorta.

\subsection{Fast ROI Analysis}

A cardiac centroid and region of interest containing the heart were calculated from the $\mathrm{H} 1$ images for each slice as follows. Firstly, in order to reduce the effect of noise and signal from non-cardiac structures, the H1 images were filtered with a smoothing filter and all pixels with magnitude less than $5 \%$ of the maximum magnitude within the $3 \mathrm{D}$ volume were set to zero. Secondly, the ROI for each slice was iteratively refined. For each iteration, the centroid of the H1 image was computed for each slice. A 3D line was then fitted to the centroids of all slices by linear least squares. A distance distribution of all H1 pixels to the 3D line was calculated and weighted with pixel's intensity values. A Gaussian curve was fitted to the distribution and all pixels greater than a certain distance from the line were removed. The 3D centroid of the H1 volume was then computed and compared to the previous $3 \mathrm{D}$ centroid. The iteration terminated when the distance between successive 3D centroids was less than one pixel. In most cases, the iteration terminated after only one loop. Finally, the ROI was adjusted in each slice by using the Gaussian fitting method again but applied to the distribution of each slice separately. The result is shown in Fig. 3. 


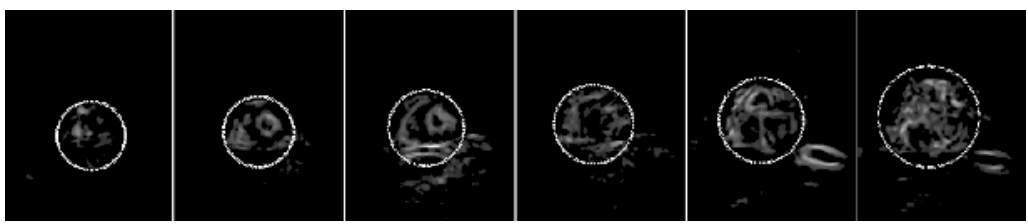

Fig. 3. Approximation of ROI in slices (apex to base from left to right)

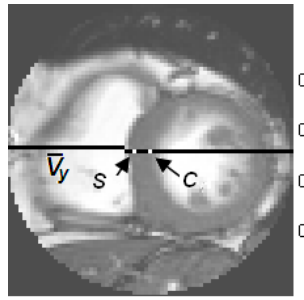

(a)

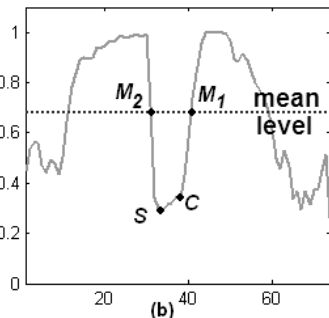

(b) 40

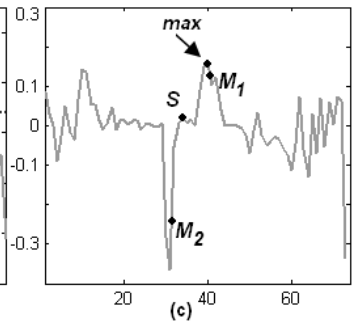

Fig. 4. Acquisition of threshold level

\subsection{Parameters for Blood Pool Segmentation}

In order to provide an initial segmentation of the LV blood pool, as well as a separation of RV and LV blood pools, we use the DC images within ROI (Fig. 4a) to locate a pixel within the septal myocardium and find the threshold level which best discriminates the blood and myocardial signals. Firstly, the midventricular SA slice closest to the $3 \mathrm{D}$ centroid was chosen. The center of the ROI was obtained by intersecting the 3D central line (from Sect. 2.3) with the slice, marked $C$ in Fig. 4. This point is almost always close to the interventricular septum. A line passing through $C$ was defined in the direction of $\bar{V}_{y}$. The intensity of the DC image along this line (Fig. 4b) was then used to locate the septum by searching for a local minimum. Once a septal point $S$ was found, the LV could be located on the $-\bar{V}_{y}$ side and the blood pool threshold could also be determined by searching for a local maximum on the gradient curve (Fig. 4k). To avoid the noise and uncertainty inherent in analyzing only a single sample, we also analyzed eight additional lines parallel to $\bar{V}_{y}$ and computed the average value of these boundary pixels.

\subsection{Detection}

The LV blood pool in the middle slice (i.e. the slice closest to the $3 \mathrm{D}$ centroid) was firstly localized on the $-\bar{V}_{y}$ side of $S$, as shown in Fig. 4a and Fig. 5 a. A convex hull (Fig. 5b) was then used to reduce the impact of the papillary muscles, as in many other papers (e.g. [5, 8] ).

To find the LV blood pools in adjacent slices, we modified the method proposed in [5]. The analysis was based on the binary images after thresholding, and assumed that the LV regions are spatially coherent between slices. The LV 


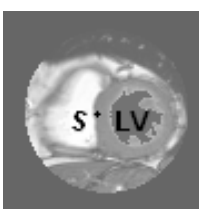

(a)

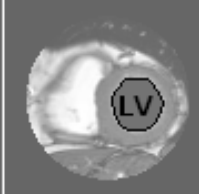

(b)

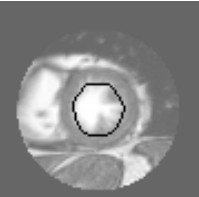

(c)

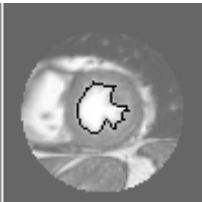

(d)

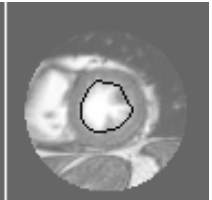

(e)

Fig. 5. Locating LV regions on the middle slice and its neighboring one

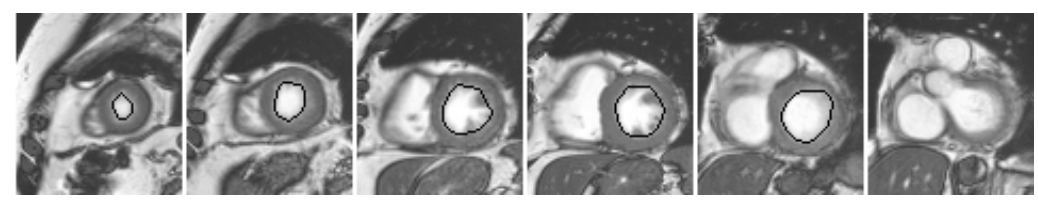

Fig. 6. Contours of the detected LV regions on slices

region detected in the middle slice was projected to its two neighboring slices and the binary objects on the neighboring slices were compared to the projected LV region. Rather than project in the direction normal to the slice [5], we projected the regions in the average long axis direction $\bar{V}_{x}$. This enables better robustness to the orientation of the image planes. After projection the most similar object in each slice was selected. The similarity of two objects was calculated by the area of the intersection divided by the area of union [5. An example is shown in Fig. 5 b-5e. Figure 5b is the middle slice with the detected LV region superimposed on it. The region is projected to its neighboring slice (Fig. 55) and the most similar object is then found (Fig. 5d). The convex hull is finally applied to the new region (Fig. 5e).

With this method, the LV regions on all slices were located (Fig. 6). However, no region would be reported if similarity criteria was violated, as in the last slice of Fig. 6. A 3D line was then fitted to the centroids of the LV regions.

\section{Results}

The fully automated method was implemented in Matlab and required approximately 4.13 seconds (not compiled) to run on a PC (Pentium IV $3.2 \mathrm{GHz}$ ) for each case, excluding the DICOM file reading time.

Only one case out of 330 failed to detect the LV, in which the RV was found instead. The remaining 329 cases were used for quantitative comparison. The orientation error of the automated method was computed by the angle between the $3 \mathrm{D}$ central line (automated $V_{x}$ ) from the method and the ground truth $V_{x}$ from the observers. This was compared to the orientation error of the manual planning in Table 1, which was obtained by the angle between the normal to the SA images and the ground truth $V_{x}$. The two errors are very similar in both observers and they are within twice the inter-observer error of $3.53 \pm 2.43$ between Observer $A$ and $B$. 
Table 1. Comparison of the orientation Errors in mean \pm std format (unit: degree)

\begin{tabular}{|l|c|c|}
\hline & $V_{x}$ in automatic method & SA normal in Manual planning \\
\hline$V_{x}$ by Observer $A$ & $6.37 \pm 4.41$ & $6.30 \pm 3.66$ \\
\hline$V_{x}$ by Observer $B$ & $6.14 \pm 4.11$ & $6.80 \pm 3.95$ \\
\hline
\end{tabular}

Table 2. Distance errors between automated method and two observers (unit: $\mathrm{mm}$ )

\begin{tabular}{|c|c|c|c|c|c|c|}
\hline & \multicolumn{2}{|c|}{ Apical Slice } & \multicolumn{2}{c|}{ Middle Slice } & \multicolumn{2}{c|}{ Basal Slice } \\
\cline { 2 - 7 } & $V_{y}$ & $V_{z}$ & $V_{y}$ & $V_{z}$ & $V_{y}$ & $V_{z}$ \\
\hline$V_{x}$ by Observer $A$ & $0.8 \pm 3.0$ & $-2.2 \pm 3.7$ & $1.2 \pm 2.6$ & $-2.6 \pm 2.4$ & $1.6 \pm 4.3$ & $-3.0 \pm 3.7$ \\
\hline$V_{x}$ by Observer $B$ & $0.5 \pm 2.9$ & $-2.7 \pm 3.3$ & $0.7 \pm 2.4$ & $-3.3 \pm 2.5$ & $0.9 \pm 3.9$ & $-3.8 \pm 4.0$ \\
\hline
\end{tabular}

We also computed the average manually determined orientations $\bar{V}_{x}, \bar{V}_{y}$ and $\bar{V}_{z}$ for each observer from the fiducial markers applied to the reference images. The inter-observer angular errors in $\bar{V}_{x}, \bar{V}_{y}, \bar{V}_{z}$ were $0.37,3.01$ and 3.03 degrees. The angular errors between $\bar{V}_{x}$ and the ground truth $V_{x}$ were $10.56 \pm 5.67$ and $10.44 \pm 5.50$ for Observer $A$ and Observer $B$. In $98 \%$ of cases, $\bar{V}_{x}$ was within 23.7 degrees of $V_{x}$, showing that the $\mathrm{LV}$ orientation is remarkably consistent across patients.

In order to compute the position errors, both the ground truth $V_{x}$ and the automated $V_{x}$ were intersected with the slices and the positions of the two intersection points were compared with each other in the ground truth coordinate system. The slices closest to the apex and base and the middle slice between these two end slices were used for the purposes of comparison. Table 2 shows the errors between the automated method and two observers in mean \pm std. format. Figure 7 shows the distribution of the errors of the worst case (Observer $A$ ). The horizontal axis represents the $V_{y}$ direction and the vertical is $V_{z}$, defined in Fig. 1]. It can be seen that the automatic results and the ground truth agree closely each other. There is a small bias in the $V_{z}$ direction which may be caused by the differences between the manual and automatic measurement methods. The
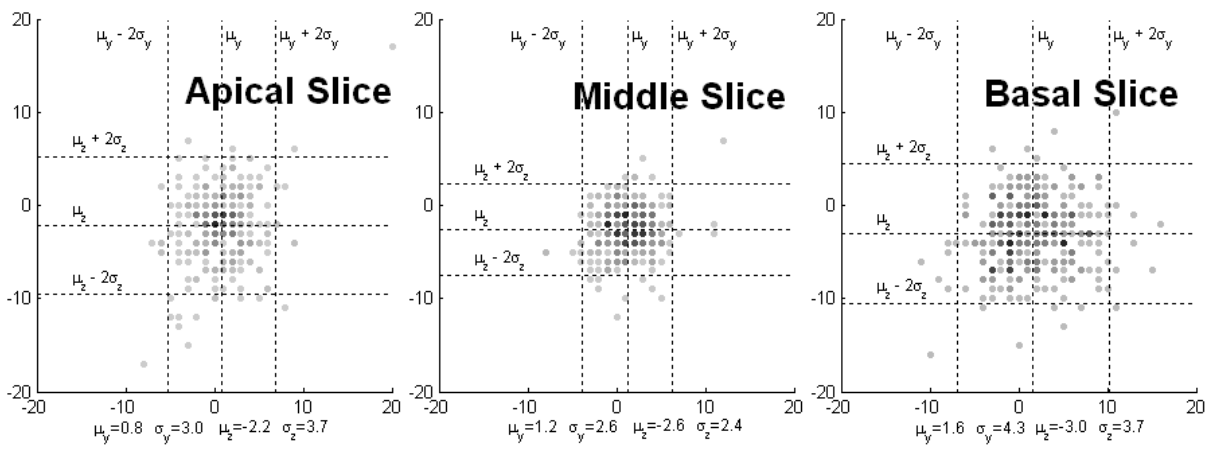

Fig. 7. Errors between ground truth $V_{x}$ (Observer $A$ ) and automatic $V_{x}$ on slices 
ground truth $V_{x}$ was measured only at end-diastole while the automatic $V_{x}$ was based on the average images.

\section{Conclusion and Discussion}

The fully automatic method presented in this paper has been proven efficient and robust for detecting the location and orientation of the LV. The errors show that the automated method is as good as the manual scan planning results performed by experienced technicians. Our method assumes that cine images have been acquired approximately orthogonal to the LV long axis, and that average cardiac orientation axes have been pre-calculated using historical data, since this is within 23.7 degrees of the ground truth axes in $98 \%$ of cases. We also assume that the images are ECG gated; however, the method could be extended to self gated acquisitions by automatic detection of the heart rate.

\section{References}

1. Santarelli, M., Positano, V., Michelassi, C., Lombardi, M., Landini, L.: Automated cardiac MR image segmentation: theory and measurement evaluation. Med. Eng. Phys. 25 (2003) 149-159

2. Lorenzo-Valdés, M., Sanchez-Ortiz, G., Mohiaddin, R., Rueckert, D.: Segmentation of $4 \mathrm{D}$ cardiac MR images using a probabilistic atlas and the EM algorithm. In: MICCAI'03. (2003) 440-450

3. Mitchell, S., Bosch, J., Lelieveldt, B., van der Geest, R., Reiber, J., Sonka, M.: 3$\mathrm{D}$ active appearance models: Segmentation of cardiac MR and ultrasound images. IEEE Trans. Med. Imag. 21(9) (2002) 1167-1178

4. Kaus, M.R., von Berg, J., Niessen, W., Pekar, V.: Automated segmentation of the left ventricle in cardiac MRI. In: MICCAI'03. (2003) 432-439

5. Montillo, A., Metaxas, D., Axel, L.: Automated segmentation of the left and right ventricles in 4D cardiac SPAMM images. In: MICCAI'02. (2002) 620-633

6. Spreeuwers, L., Breeuwer, M.: Automatic detection of the myocardial boundaries of the right and left ventricle. SPIE: Med. Imag. 4322 (2001) 1207-1217

7. Danilouchkine, M., Westenberg, J., Reiber, J., Lelieveldt, B.: Accuracy of shortaxis cardiac MRI automatically derived from scout acquisitions in free-breathing and breath-holding modes. MAGMA 18 (2005) 7-18

8. Jackson, C., Robson, M., Francis, J., Noble, J.: Automatic planning of the acquisition of cardiac MR images. In: MICCAI'03. (2003) 541-548

9. Anderson, C.: Rationale and design of the cardiac magnetic resonance imaging substudy of the ONTARGET trial programme. J. Int. Med. Res. 33(4) (2005) $50 \mathrm{~A}-57 \mathrm{~A}$

10. Young, A., Cowan, B., Thrupp, S., Hedley, W., Dell'Italia, L.: Left ventricular mass and volume: fast calculation with guide-point modeling on MR images. Radiology 216 (2000) 597-602

11. Sörgel, W., Vaerman, V.: Automatic heart localization from 4D MRI datasets. SPIE: Med. Imag. 3034 (1997) 333-344

12. Gering, D.: Automatic segmentation of cardiac MRI. In: MICCAI'03. (2003) $524-532$ 